\title{
Effect of Probe Channeling on Differential Phase Contrast at the Atomic Scale
}

\author{
Soohyun $\operatorname{Im}^{1}$, Jared Johnson ${ }^{1}$, and Jinwoo Hwang ${ }^{1}$ \\ 1. Department of Materials Science and Engineering, The Ohio State University, Columbus, OH 43212
}

Direct quantification of atomic scale electromagnetic structures can potentially lead to a tremendous impact to the characterization of functional materials. Recent experiments showed that differential phase contrast (DPC) imaging [1] performed in an aberration corrected STEM may be able to detect the internal fields around individual atoms [2]. However, the exact understanding of atomic scale DPC mechanism is still lacking. The current understanding involves the perturbation in the incoming electron wave by the atomic potential, which leads to the re-distribution of intensity in the ronchigram [3]. Since the magnitude of the intensity re-distribution may be small, it has been generally agreed that the DPC signal can only be reliable only when weak phase approximation is valid (i.e. sample is very thin). However, how to set the practical limit of sample thickness for DPC remains unknown. Also, while the effect of probe channeling has been studied for Z-contrast imaging [e.g. 4, 5], its effect to DPC has not been known. In the present work, we show that the atomic scale DPC signals strongly depend on probe channeling oscillation and sample thickness. In particular, we show that the internal field information is directly related to the $2^{\text {nd }}$ derivative of the probe channeling oscillation.

We use multislice algorithm to simulate DPC images with a segmented detector with 4 quadrants (Fig. 1a), same as our experimental settings in FEI Titan STEM. By calculating the differences between X1 and $\mathrm{X} 2$, and $\mathrm{Y} 1$ and $\mathrm{Y} 2$ images, the field vectors around each atom can be calculated [1, 2]. The simulated STEM CBED pattern from a point near the Sr column shows re-distribution of intensity inside the zero beam (Fig. 1b), consistent to Müller et al [3]. The CBED pattern also provides guidance on the optimum detection angle. We used the inner angle of $8.8 \mathrm{mrad}$ in this simulation, but we also found that $5 \mathrm{mrad}$ inner angle provides higher vector magnitudes without altering the vector angles. We also found that, for samples that are thin enough (less than $\sim 10 \mathrm{~nm}$ thick), the overlapping diffraction disks (e.g. Fig. 1c) do not significantly affect DPC signals. This may be because, in cubic materials such as $\mathrm{SrTiO}_{3}$, the diffraction patterns are highly symmetric and therefore the diffraction intensities are uniformly distributed on each segment of the detector. We also found that including thermal vibration in the simulation does not significantly influence the DPC signals.

However, we found that probe channeling oscillation and sample thickness significantly affect DPC signals. Figure 2 shows that the internal field vectors change as a function of sample thickness. In particular, at certain thicknesses $(e . g .3 .12 \mathrm{~nm})$ the vector directions become completely opposite to the original directions. We found that the "switching" of the direction happens almost precisely at the inflection points of the probe oscillation function (blue line) along the atomic column (Fig. 3). Since the switching of the direction happens gradually (i.e. vectors become smaller before switching), the mean vector magnitude in each image as a function of thickness (black line) also shows similar oscillation. The magnitude oscillation also shows overall increase as a function of thickness (red line), which must reflect a simple mass dependence. The results suggest a strong dependence of atomic scale DPC signals on probe channeling, which provides an important step toward the quantification of atomic scale electromagnetic fields in functional materials. We will also further discuss the theoretical understanding of the DPC's dependence on probe channeling, and the comparison to experimental results. 


\section{References}

[1] H. Rose, Ultramicroscopy, 2, 251 (1976).

[2] N. Shibata et al., Nat. Phys., 8, 611 (2012).

[3] K. Müller et al., Nat. Commun., 5, 5653 (2014).

[4] P. M. Voyles, D. A. Muller, and E. J. Kirkland, Microsc. Microanal., 10, 291 (2004)

[5] J. Hwang et al., Phys. Rev. Lett., 111, 266101 (2013).
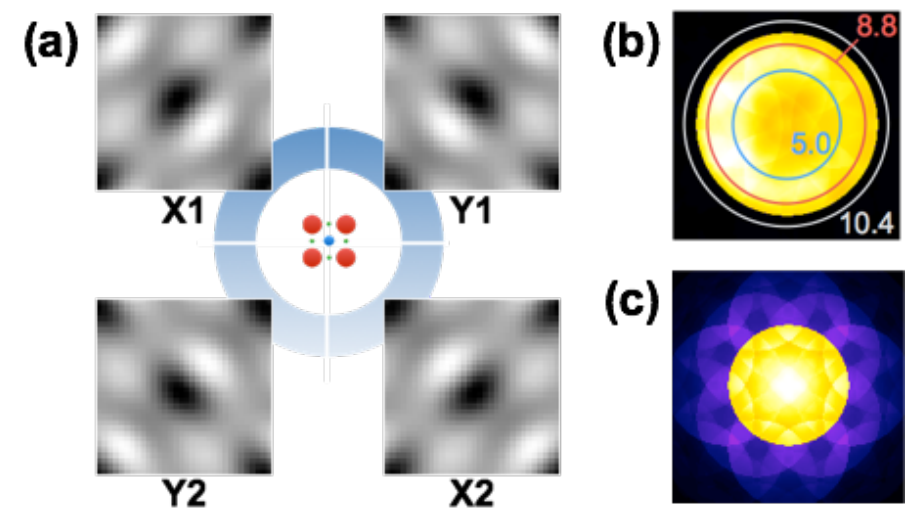

Figure 1. (a) Images simulated for $\mathrm{SrTiO}_{3}$ on a segmented detector. (b) CBED pattern simulated for a position near the Sr column shows redistribution of intensity inside the zero beam with $9.6 \mathrm{mrad}$ convergence half angle. The concentric circles and numbers indicate the detector inner angles (5.0 and 8.8 $\mathrm{mrad})$, and outer angle (10.4 mrad) used in the simulation. (c) PACBED pattern simulated for $9.37 \mathrm{~nm}$ thick $\mathrm{SrTiO}_{3}$.

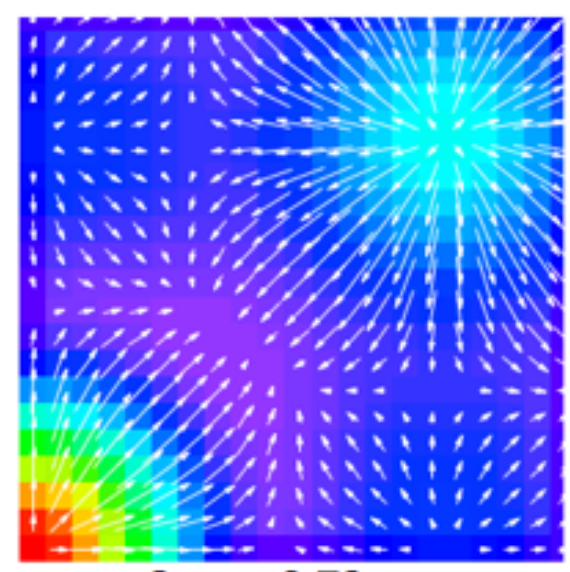

2 u.c. $0.78 \mathrm{~nm}$

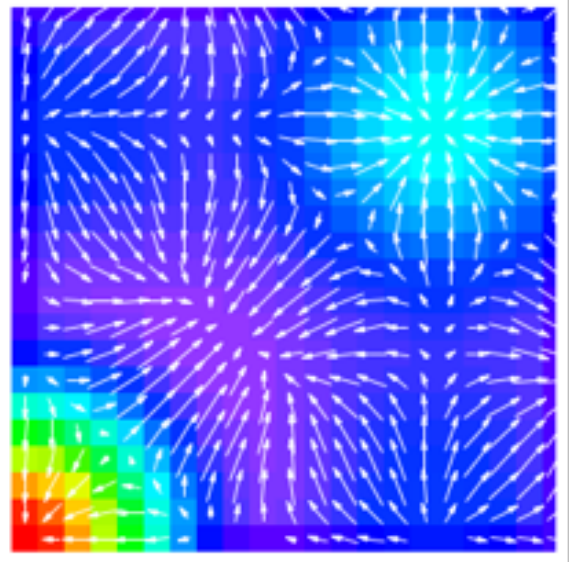

8 u.c. $3.12 \mathrm{~nm}$

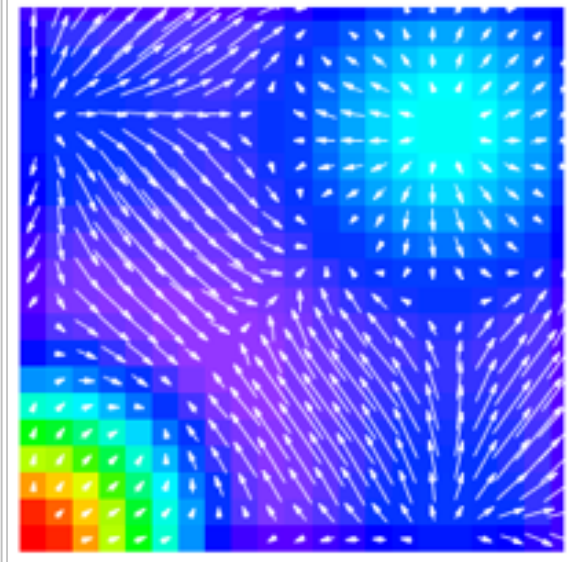

24 u.c. $9.37 \mathrm{~nm}$

Figure 2. (a) Simulated DPC images showing field vectors around $\mathrm{Sr}$ and $\mathrm{Ti}$ columns in $\mathrm{SrTiO}_{3}$, with sample thickness from 0.78 to $9.37 \mathrm{~nm}$. The vector magnitudes are normalized for visual clarity.

Figure 3. (a) Probe channeling oscillation simulated at the $\mathrm{Sr}$ column as a function of sample thickness. (b) Probe oscillation compared to the absolute mean vector magnitude of each image as a function of sample depth. (c) The open and solid green arrows indicate the relative directions of the field near the atomic columns. The field direction switches at the inflection points of the graph.
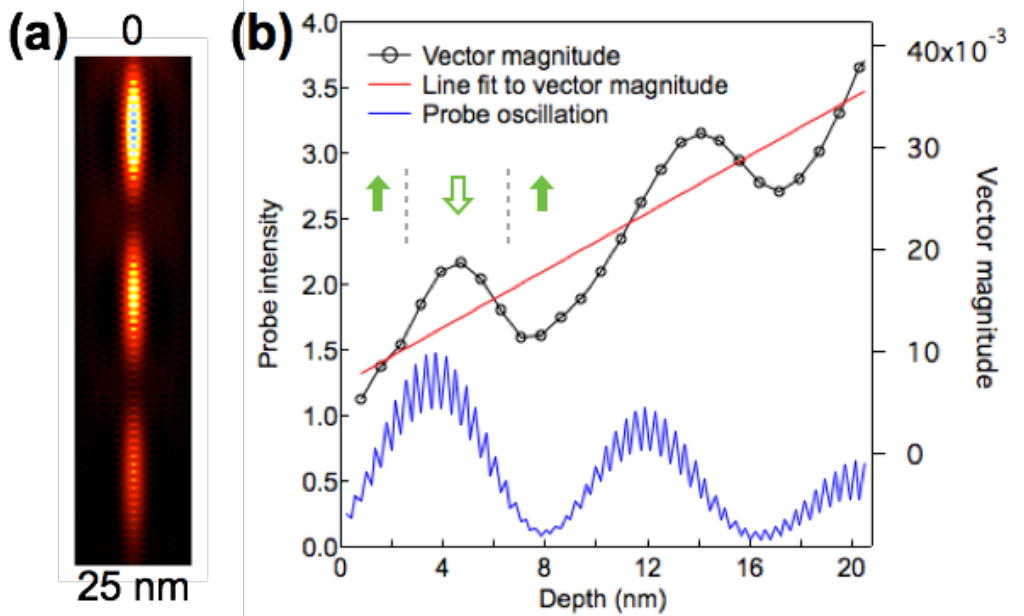